\title{
Eine Porphinsynthese durch anodische Oxydation von Pyrrol in Anwesenheit von Aldehyden *
}

\author{
A. Stanienda \\ 2. Chemisches Institut der Humboldt-Universität, Berlin \\ (Z. Naturforschg. 22 b, 1107-1111 [1967] ; eingegangen am 12. April 1967)
}

\begin{abstract}
Pyrrole has been oxidized in an irreversible wave on a rotating Pt-disc electrode in acetonitrile. When oxidation was carried out in the presence of benzaldehyde it was possible to isolate tetraphenylporphine. The porphine synthesis is initiated by protons formed on the oxidation of pyrrole. Addition and condensation of protonated pyrrole molecules with benzaldehyde formed tetraphenyl porphinogen, which was oxidized to tetraphenylporphine anodically or by redox reaction in solution. This redox reaction with pyrrole forms dihydropyrrole which proceeds finally to chlorins and explain also the current yield greater than a hundred per cent. This synthesis of porphine can be achieved in a few minutes at $-70^{\circ} \mathrm{C}$ by addition of acid to a solution of pyrrole and benzaldehyde.
\end{abstract}

Nach 10-stdg. Einwirkung von $\gamma$-Strahlen $(0,5$ Megaröntgen/Stde.) auf eine Lösung von Pyrrol und Benzaldehyd in Pyridin oder Wasser erhielt Szutka ${ }^{1}$ meso-Tetraphenylporphin in einer Ausbeute $\ll 1 \%$ bezogen auf Pyrrol. Der Autor sieht in dieser Umsetzung eine Modellreaktion für eine Porphinbildung in einem sehr frühen Stadium der erdgeschichtlichen Entwicklung. Präparativ erhält man meso-Tetraphenylporphin nach RoTHEMUnd ${ }^{2}$ durch zweitägiges Erhitzen von Pyrrol und Benzaldehyd in Pyridin im Einschlußrohr auf 180 bis $200^{\circ} \mathrm{C}$. Dieser Reaktionsmechanismus ist von BADGER, Jones und Lasletr ${ }^{3}$ durch Isolierung eines Dipyrromethen aufgeklärt worden, wobei die Bildung von Chlorinen auf Redoxvorgänge in der Lösung zurückgeführt wird. Da sich die Reaktionsbedingungen der Porphinbildung nach Rothemund und SzutKa so sehr unterscheiden, wird auch der Reaktionsmechanismus bei beiden Reaktionen unterschiedlich ablaufen. Zwischen photochemischen Reaktionen, die durch eine ionisierende Strahlung ausgelöst werden und anodischen Oxydationen bestehen gewisse Analogien **, daher konnte erwartet werden, daß Tetraphenylporphin auch bei der anodischen Oxydation von Pyrrol in Gegenwart von Benzaldehyd gebildet wird. In der vorliegenden Mitteilung wird über eine solche Porphinbildung, die auch bei $-70^{\circ} \mathrm{C}$ in wenigen Min. abläuft, berichtet und der Mechanismus disku-

* Der wesentliche Inhalt wurde auszugsweise auf dem IV. Internationalen polarographischen Kongreß vom 4. 7. bis 8. 7. 1966 in Prag vorgetragen.

1 A. Szutka, Rad. Res. 19, 183 [1963] ; A. Szutka, in: „The origins of prebiological systems and their molecular matrices“, ed. by S. W. Fox, Academic Press Inc., New York 1963. - A. Szutka u. R. H. Radzilowski, Z. Naturforschg. 20 b, 624 [1965]. tiert, der auch für die Synthese unter der Einwirkung von Strahlung zutreffen dürfte.

\section{Ergebnisse und Diskussion}

Pyrrol wird in Acetonitril an einer rotierenden Platin-Scheibenelektrode in einer mehrelektronigen, irreversiblen Stufe oxydiert, die bei höherer Konzentration an Pyrrol $\left(>2 \cdot 10^{-4} \mathrm{Mol} / l\right)$ in eine Vorund eine Hauptstufe zerfällt. Nach Erreichen des Grenzstrombereiches tritt als Folge einer Filmbildung ein starker Stromabfall auf (Abb. 1). Reproduzierbare Messungen konnten nur nach Abschmirgeln der Elektrodenoberfläche erhalten werden, denn schon nach der ersten Aufnahme einer Stromspannungskurve war die Oberfläche mit einer braunen

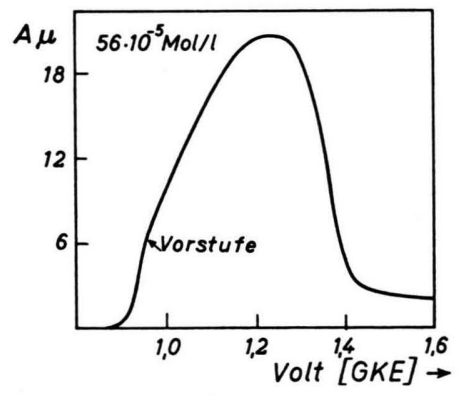

Abb. 1. Stromspannungskurve von Pyrrol an einer rotierenden Pt-Scheibenelektrode $(U=675 /$ Min., $v=0,0058 \mathrm{~V} / \mathrm{s})$.

2 P. J. Rothemund, J. Amer. chem. Soc. 57, 2010 [1935] ; 61, 2912 [1939].

3 G. M. Badger, R. A. Jones u. R. L. Laslett, Austral. J. Chem. 17, 1028 [1964].

* Zum Beispiel lineare Beziehungen zwischen Photoionisations-Potentialen und anodischen Halbstufenpotentialen bei $\pi$-Elektronensystemen. 
Schicht bedeckt. Im gemessenen Konzentrationsbereich von ca. $10^{-5}$ bis $10^{-3} \mathrm{Mol} / l$ ist die Stufenhöhe Konzentrations-proportional (Tab. 1), das Halbstufenpotential nach $E_{1 / 2}=0,804-0,10 \cdot \log C$ abhängig von der Konzentration $C$. Die nicht lineare Abhängigkeit der Stufenhöhe von der Kreisfrequenz der Elektrode ist auf eine dem Elektronendurchtritt nachfolgende chemische Reaktion zurückzuführen, deren Endprodukt unbekannt ist (Abb. 2). Eine Zugabe von Benzaldehyd ist ohne wesentlichen Einfluß auf das Halbstufenpotential oder die Stufenhöhe. In

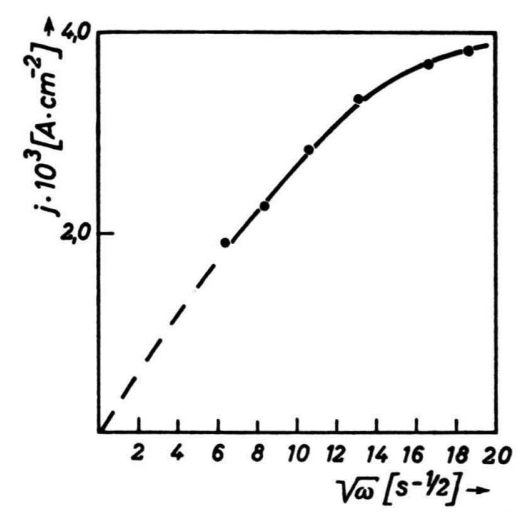

Abb. 2. Abhängigkeit der Stufenhöhe von Pyrrol ( $C=$ $\left.5 \cdot 10^{-5} \mathrm{~mol} / l\right)$ von $V \omega$.

Gegenwart von Pyridin sinkt die Höhe der Oxydationsstufe von Pyrrol ab. Dies ist offenbar auf eine Filmbildung zurückzuführen, die schon vor Erreichen des Grenzstrombereiches die Elektrodenoberfläche bedeckt. Das folgt aus einer coulometrischen Elektrolyse bei einem kontrollierten Potential von

\begin{tabular}{cccc}
\hline $\begin{array}{c}C \cdot 10^{5} \\
\mathrm{Mol} / l\end{array}$ & $\begin{array}{c}E_{1} \mathrm{l}_{2} \\
\text { Volt }\end{array}$ & $\begin{array}{c}E_{1} / 2 \\
\text { berechnet } \\
\text { nach* }\end{array}$ & $\frac{i \cdot 10^{5}}{C \cdot K}\left(\mathrm{~A} \cdot \mathrm{cm}^{3} \cdot \mathrm{Mol}^{-1}\right)$ \\
\hline 2 & 1,27 & 1,27 & 3,77 \\
4 & 1,24 & 1,24 & 3,84 \\
8 & 1,21 & 1,21 & 3,60 \\
16 & 1,18 & 1,18 & 3,50 \\
36 & $1,12 / 1,22 * *$ & 1,15 & 3,63 \\
96 & 1,08 & 1,10 & 3,64
\end{tabular}

Tab. 1. Konzentrations-Abhängigkeit von Stufenhöhe und Halbstufenpotential der Oxydationsstufe von Pyrrol in 0,1-m. $\mathrm{LiClO}_{4} / \mathrm{CH}_{3} \mathrm{CN}$. Gegenelektrode: Gesättigte Kalomelelektrode (GKE). ${ }^{*} E_{1 / 2}=0,804-0,10 \cdot \log C .{ }^{* *}$ Auftrennung der Stufe in Vor- und Hauptstufe.
$K=0,62 \cdot n \cdot F \cdot q \cdot D^{2 / 3} \cdot v^{-1 / 6} \cdot \omega^{1 / 2} \equiv 1$.

${ }^{4}$ A. Treirs u. H. G. Kolm, Liebigs Ann. Chem. 606, 166 [1957].

5 G. F. SмIтн, in :„Advances in Heteracyclic Chemistry“, Bd. 2, S. 287. Herausgeber: A. R. Katritzky, Academic Press, New York u. London.
1,3 Volt (GKE), bei der die Differenz in der umgesetzten Elektrizitätsmenge zwischen der reinen Pyrrollösung und der mit Pyridin versetzten nur - 1\% betrug, bei Anwesenheit von Benzaldehyd -3 Prozent.

Bei der anodischen Oxydation von Pyrrol an einer großflächigen Pt-Netzelektrode bei einem kontrollierten Potential von 1,0 Volt in Gegenwart von Benzaldehyd kann neben Verharzungsprodukt Tetraphenylporphin isoliert werden. Wie Tab. 2 zeigt, wird bei Anwesenheit von H-Ionen-Akzeptoren eine Porphinsythese verhindert. Daraus kann gefolgert werden, da $\beta$ bei der Elektrolyse Protonen entstehen, die an der Reaktion teilnehmen. Der Protonenakzeptor kann nicht mit Sicherheit angegeben werden. In der Literatur besteht über die Basizität von Pyrrol eine Meinungsverschiedenheit. Nach Treibs und $\mathrm{KoLM}^{4}$ ist Pyrrol in verdünnter wäßriger Salzsäure leicht oxydabel, liegt aber bei Ausschluß von Sauerstoff protoniert vor. Dies ist nach $\mathrm{S}_{\text {мiтн }}{ }^{5}$ mit gemessenen $p_{\mathrm{K} a}$-Werten von $-1,7$ bis $0,3^{6}$ bzw. $-3,8^{7}$ unvereinbar. In Acetonitril wurde der $p_{\mathrm{K}}$-Wert von Pyrrol noch nicht gemessen.

Wie oben gezeigt wurde, erfolgt die anodische Oxydation von Pyrrol in einer mehrelektronigen $(n=3,5)$, irreversiblen Stufe, wobei Protonen frei werden, die nur vom Pyrrol angelagert werden können. Wäre eine solche Anlagerung nicht möglich, dann würde eine mehrelektronige Oxydation vermutlich nicht in einer Stufe ablaufen. Außerdem wäre bei Anwesenheit eines H-Ionen-Akzeptors (Pyridin) ein größerer Unterschied in der umgesetzten Elektrizitätsmenge oder in der Lage der Stufe zu erwarten. Die Bildung von Tetraphenylporphin kann sowohl mit Pyrrol (a) als auch mit Benzaldehyd als Protonenakzeptor (b) verstanden werden:

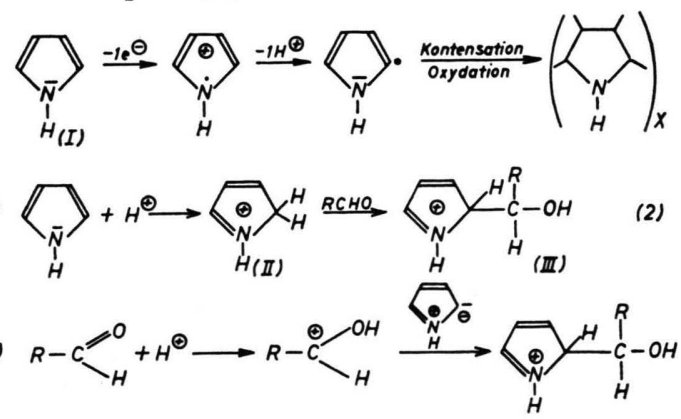

(1)

- H. Narqui u. Q. Fernanda, J. org. Chemistry 25, 551 [1960].

7 Y. Chang u. E. B. Whipple, J. Amer. chem. Soc. 85, 2763 [1963]. 

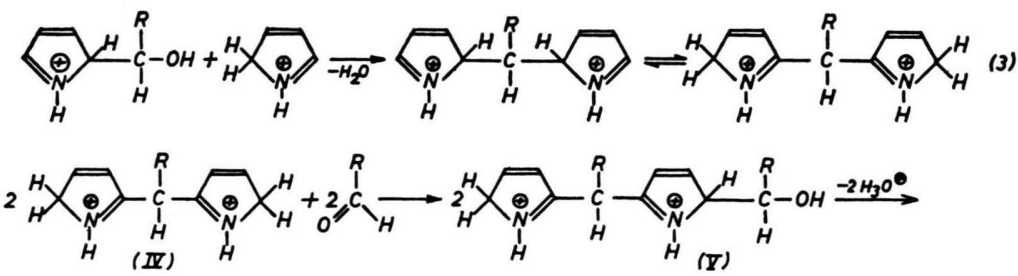

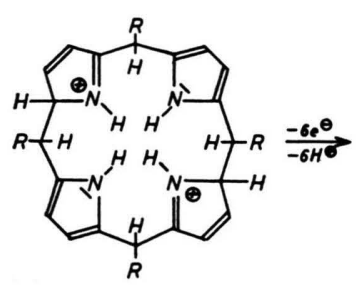

(II)

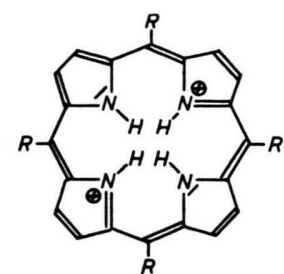

(EII)
Aus obigem Formelschema geht hervor, daß zunächst Pyrrolradikalkationen entstehen, die Protonen abspalten und dann polymerisieren, an Redoxreaktionen teilnehmen und schließlich verharzen. Die abgespaltenen Protonen lösen die angegebene Reaktionsfolge aus. Zur Bildung einer Tetraphenylporphinogen-Molekel( VI) werden 4 Protonen benötigt. Das bei der Reaktion entstehende Wasser ist in Acetonitril eine starke Base $\left(K_{\mathrm{H}_{3} \mathrm{O}}{ }^{\oplus}=5 \cdot 10^{-3}\right)^{8}$ und man kann daher annehmen, daß bei der Reaktion $\mathrm{V} \rightarrow \mathrm{VI}$ nicht $\mathrm{H}_{2} \mathrm{O}$, sondern $\mathrm{H}_{3} \mathrm{O}^{\oplus}$ abgespalten wird. Bei der nun folgenden sechselektronigen Oxydation zum Porphin $(\mathrm{VI} \rightarrow \mathrm{VII})$ werden mindestens 6 Protonen frei, die weitere Pyrrolmoleküle in den reaktionsbereiten Zustand bringen. Zwei Protonen werden vom basischen Porphinmolekül zurückgehalten, wodurch es vor Oxydation geschützt wird ${ }^{*}$. Die Berechnung der Stromausbeute erfolgte nach:

$$
G=\frac{i \cdot t \cdot M}{6.96500} \quad M=620=\text { Mol.-Gew. von VI }
$$

Dabei wurde der 4-Elektronenübergang, der zur Erzeugung der die Reaktion einleitenden 4 Protonen notwendig ist, vernachlässigt, weil bei der Reaktion auch Protonen entstehen und zwar nach dem Bildungsgesetz einer geometrischen Reihe: $a_{\mathrm{n}}=$ $4 \cdot 1,5^{n-1}$. Allerdings werden nicht alle so erzeugten Protonen für die Porphinsynthese wirksam, sondern teilweise von der bei der Reaktion entstehen-

8 I. M. Kolthoff u. S. Ineda, J. physic. Chem. 65, 1020 [1961].

* Durch Anlagerung von Protonen an freie Elektronenpaare von N-Atomen im Porphin wird das anodische Halbstufenpotential beträchtlich erhöht. den Base $\mathrm{H}_{2} \mathrm{O}$ abgefangen. Auf diese anwesenden Protonen ist wahrscheinlich auch der autokatalytische Effekt zurückzuführen, der nach Szutka auch nach Beendigung der Bestrahlung der Reaktionslösung eine weitere Porphinbildung ermöglicht ${ }^{1}$.

Die gemessene Stromausbeute von über $100 \%$ ist nur dadurch zu erklären, daß unter den Reaktionsbedingungen, durch das Spülen der Lösung mit Argon, die Reaktionspartner sofort von der Elektrode entfernt werden und die Reaktionen außerhalb der näheren Elektrodenumgebung stattfinden. Bei den Redoxreaktionen ist Pyrrol Elektronenakzeptor, wodurch Dihydropyrrol entsteht, das an den Reaktionen mit Benzaldehyd teilnimmt und so zu Chlorinen führt. Die Ausbeute an Chlorin kann erheblich gesteigert werden durch Erzeugung einer sterischen Hinderung in der Porphin-Molekel. Dies kann erreicht werden, wenn als Aldehyd-Komponente ortho-Chlorbenzaldehyd genommen wird **. Nach Woodward ${ }^{9}$ ist bei raumfüllenden Substituenten in den meso-Stellungen die tetragonale Konfiguration in $\beta$-Stellung des Pyrrolkernes vor der trigonalen bevorzugt.

Wie Tab. 2 zeigt, steigt die Ausbeute an Porphin, wenn die Elektrolysezeit verkürzt wird ( $\mathrm{Nr} .1-5)$, weil dann weniger Protonen frei werden, die Nebenreaktionen einleiten, die schneller ablaufen als die Synthese von Tetraphenylporphin. Auch eine gleichmäßige Erhöhung der Konzentration von Pyrrol und Benzaldehyd führt zu einer größeren Stromausbeute besonders dann, wenn $i \cdot \boldsymbol{t}$ konstant gehalten wird (Nr. 3, 4 und 5), weil dadurch ein günstigeres Konzentrations-Verhältnis [Protonen]/[Pyrrol] erreicht wird. Der Einfluß von Basen wird durch Vergleich von Nr. 4 mit 6, 7 und 8 deutlich. Bei einer Erhöhung nur der Konzentration von Benzaldehyd sinkt die Ausbeute, weil dann Pyrrol offenbar mit

** Bisher unveröffentlichte Versuche.

9 R. B. Wodward, Angew. Chem. 72, 651 [1960]. 


\begin{tabular}{|c|c|c|c|c|c|c|}
\hline Nr. & $\begin{array}{l}\text { Pyrrol } \\
\text { [mMol }]\end{array}$ & $\begin{array}{l}\text { Benz- } \\
\text { aldehyd } \\
{[\mathrm{mMol}]}\end{array}$ & $\begin{array}{c}\text { Tetraphenyl- } \\
\text { porphin } \\
{[\mathrm{mMol}]}\end{array}$ & $\begin{array}{c}i \cdot t \\
{[\mathrm{~A} \cdot \mathrm{s}]}\end{array}$ & $\begin{array}{c}\text { Strom- } \\
\text { ausbeute } \\
{[\%]}\end{array}$ & Bemerkungen \\
\hline 1 & 0,72 & 0,50 & $2 \cdot 10^{-4}$ & 23,3 & 6,0 & \multirow{11}{*}{$\begin{array}{l}T=183^{\prime} \\
T=36^{\prime} \\
T=15^{\prime} \\
T=15^{\prime} \\
T=15^{\prime} \\
+0,072 \mathrm{mMol} \\
\text { Triäthylamin } \\
+0,72 \mathrm{mMol} \\
\text { Triäthylamin } \\
+2,5 \mathrm{mMol} \\
\text { Pyridin }\end{array}$} \\
\hline 2 & 0,72 & 0,50 & $5,9 \cdot 10^{-4}$ & 4,3 & 9,4 & \\
\hline 3 & 0,72 & 0,50 & $5,7 \cdot 10^{-4}$ & 1,7 & 23,4 & \\
\hline 4 & 1,44 & 0,99 & $23,8 \cdot 10^{-4}$ & 1,8 & 75,5 & \\
\hline 5 & 2,88 & 1,98 & $45,0 \cdot 10^{-4}$ & 1,9 & 136,0 & \\
\hline 6 & 1,44 & 0,99 & $4,5 \cdot 10^{-4}$ & 15,9 & 1,9 & \\
\hline 7 & 1,44 & 0,99 & 0 & 3,8 & 0 & \\
\hline 8 & 2,88 & 1,98 & 0 & 6,2 & 0 & \\
\hline 9 & 0,72 & 0,99 & $0,14 \cdot 10^{-4}$ & 9,5 & 1,0 & \\
\hline 10 & 0,72 & 1,98 & 0 & 4,1 & 0 & \\
\hline 11 & 1,44 & 0,50 & $0,15 \cdot 10^{-4}$ & 4,0 & 2,4 & \\
\hline
\end{tabular}

Tab. 2. Abhängigkeit der Porphinbildung bei Zimmertemperatur von der Konzentration an Pyrrol und Benzaldehyd und von der Anwesenheit von Basen, $T=$ Dauer der Elektrolyse. Lösungsmittel: 0,1-m. $\mathrm{LiClO}_{4} / \mathrm{CH}_{3} \mathrm{CN}$, Potential: 1,0 Volt [GKE]. * Der Elektrolyse wurde jeweils nur ein geringer Anteil des eingesetzten Pyrrols unterworfen. Bei der Untersuchung der Konzentrations-Abhängigkeit (Nr. 3, 4 und 5) wurde versucht, durch Änderung des kontrollierten Potentials einen annähernd gleichen Umsatz an Elektrizitätsmenge zu erreichen.

mehreren Molekülen Benzaldehyd reagieren kann (vgl. 9 und 10 mit 3), nach:

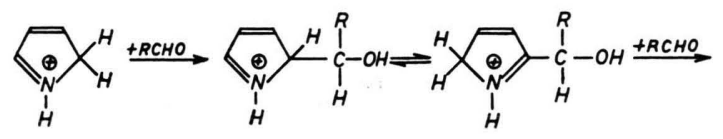<smiles>[R]Cc1ccc(C([2H])O)[nH]1</smiles>

$\mathrm{Zu}$ einer Ausbeuteverminderung führt andererseits auch eine Erhöhung der Pyrrolkonzentration, wenn die Menge an Benzaldehyd nicht auch gleichmäßig erhöht wird (vgl. 11 und 3).

Wenn der angegebene anodische Oxydationsmechanismus zutrifft, sollte es möglich sein, die elek- trochemische Erzeugung von Protonen zu umgehen und Tetraphenylporphin aus Pyrrol und Benzaldehyd durch Zugabe von Säure zu synthetisieren. Die Versuche sind in Tab. 3 zusammengestellt.

Aus diesen Ergebnissen folgt, daß die hier angegebenen Reaktionsbedingungen für eine Bildung des Porphingerüstes den physiologischen Bedingungen sehr viel ähnlicher sind, als Synthesen durch intensive Bestrahlung oder durch längeres Erhitzen auf höhere Temperatur, die der Zelle nicht zur Verfügung stehen (wohl aber $p_{\mathrm{H}}$-Änderungen und Redoxreaktionen). Unter geeigneten Bedingungen $\left(p_{\mathrm{H}}\right.$, Temperatur und Konzentration) dürfte mit Sicherheit eine wesentlich höhere Ausbeute an Porphin erreichbar sein.

\begin{tabular}{|c|c|c|c|c|c|c|c|c|}
\hline Nr. & $\begin{array}{l}\text { Pyrrol } \\
{[\mathrm{mMol}]}\end{array}$ & $\begin{array}{l}\text { Benz- } \\
\text { aldehyd } \\
{[\mathrm{mMol}]}\end{array}$ & $\begin{array}{l}\mathrm{HClO}_{4} \\
{[\mathrm{mMol}]}\end{array}$ & $\begin{array}{c}t \\
{\left[{ }^{\circ} \mathrm{C}\right]}\end{array}$ & $\begin{array}{c}\text { Tetraphenyl- } \\
\text { porphin } \\
{[\mathrm{mMol}]}\end{array}$ & $\begin{array}{c}\text { Ausbeute } \\
\text { bezogen } \\
{[\%]}\end{array}$ & $T$ & $\begin{array}{l}\text { Oxydationsmittel } \\
{[\mathrm{mMol}]}\end{array}$ \\
\hline 12 & 1,44 & 0,99 & 0,10 & -35 & $1,2 \cdot 10^{-3}$ & 0,35 & $60^{\prime}$ & $0,37 \mathrm{PbO}_{2}$ \\
\hline 13 & 1,44 & 0,99 & 0,53 & $-50 *$ & $4,0 \cdot 10^{-3}$ & 1,1 & $900^{\prime}$ & $0,37 \mathrm{PbO}_{2}$ \\
\hline 14 & 1,44 & 0,99 & 10,6 & -35 & 0 & 0 & $30^{\prime}$ & $0,37 \mathrm{PbO}_{2}$ \\
\hline 15 & 1,44 & 0,99 & 1,06 & +10 & $0,7 \cdot 10^{-3}$ & 0,2 & $5+5^{\prime * *}$ & $1,0 \quad \mathrm{FeCl}_{3}$ \\
\hline 16 & 1,44 & 0,99 & 1,06 & -20 & $3,8 \cdot 10^{-3}$ & 1,0 & $5+5^{\prime} * *$ & $1,0 \quad \mathrm{FeCl}_{3}$ \\
\hline 17 & 1,44 & 0,99 & 1,06 & $-60 *$ & $5,0 \cdot 10^{-3}$ & 1,3 & $5+5^{\prime}$ & $1,0 \quad \mathrm{FeCl}_{3}$ \\
\hline 18 & 2,88 & 1,98 & 1,06 & -20 & $7,4 \cdot 10^{-3}$ & 1,0 & $5^{\prime}$ & $2,2 \quad \mathrm{~J}_{2}$ \\
\hline
\end{tabular}

Tab. 3. Abhängigkeit der Porphinbildung in Acetonitril von der Säurekonzentration, der Temperatur $t$ und der Dauer $T$ der Reaktion. * Der Säurezusatz zur Reaktionslösung aus Pyrrol und Benzaldehyd in Acetonitril (Schmp. $\left.-39^{\circ} \mathrm{C}\right)$ erfolgte bei $-35^{\circ} \mathrm{C}$, danach wurde sofort auf -50 bzw. $-60^{\circ} \mathrm{C}$ abgekühlt und nach $T$ Min. in wäßrige Sodalösung gegeben. ${ }^{*} 5+5^{\prime}$ heißt, daß die Zugabe des Oxydationsmittels 5Min. nach dem Säurezusatz erfolgte, 5 Min. danach wurde aufgearbeitet. 


\section{Experimenteller Teil}

Die Elektrolysen wurden potentiostatisch durchgeführt, wobei die umgesetzte Elektrizitätsmenge mit einem Coulometer nach EHLERS und SEASE ${ }^{10}$ gemessen wurde. Als Leitelektrolyt diente eine 0,1-m. $\mathrm{LiClO}_{4} /$ Acetonitril-Lösung. Der Anodenraum war durch Diaphragmen vom Gegenelektroden- und Bezugselektrodenraum getrennt. Nach durchgeführter Oxydation wurde die Lösung sofort in wäßrige Sodalösung gegossen, mit Chloroform geschüttelt, danach wurde über $\mathrm{Na}_{2} \mathrm{SO}_{4}$ ge-

10 V. B. Ehlers u. J. W. Sease, Analytic. Chem. 26, 513 [1954]. trocknet und eingeengt, anschließend zwei- bis drei Mal über eine Säule aus $\mathrm{Al}_{2} \mathrm{O}_{3}$ geschickt. Die Reinheitsprüfung erfolgte durch Dünnschichtchromatographie ${ }^{11}$. Die Ausbeute wurde spektroskopisch bestimmt $\left(\varepsilon_{515}=\right.$ $\left.18,7 \cdot 10^{3} \cdot l \cdot \mathrm{Mol}^{-1} \cdot \mathrm{cm}^{-1}\right)^{1}$. Dünnschichtchromatogramm und Absorptionsspektrum stimmten mit einer Vergleichsprobe von Tetraphenylporphin völlig überein, ebenso der $\mathrm{Zn}$ (II)-Komplex.

Frl. G. BIEBl danke ich herzlich für die sorgfältige Durchführung der chromatographischen Reinigungen.

11 R. W. Balek u. A. Szutka, J. Chromatogr. 17, 127 [1965].

\title{
Uber die Temperaturabhängigkeit enzymatischer Reaktionen II * Hydrolyse von Glycerinestern durch Pankreaslipase (Glycerinesterhydrolase [3.1.1.3.])
}

\author{
G. TALSKY
}

Institut für Technische Chemie der TH München

(Z. Naturforschg. 22 b, 1111-1118 [1967]; eingegangen am 28. April 1967)

\begin{abstract}
Die Hydrolyse von Tributyrin und Triacetin durch Pankreaslipase wurde unter verschiedenen Reaktionsbedingungen untersucht, die $p_{\mathrm{H}}$-Abhängigkeit sowie $K_{\mathrm{m}}$ und $V_{\mathrm{max}}$ ermittelt. Einflüsse auf das anomale Temperaturverhalten der Reaktionsgeschwindigkeit wurden geprüft und diskutiert. Das Temperaturverhalten kann in allen Fällen als Änderung der Reaktionsverhältnisse gedeutet werden. Im Gegensatz dazu wies die Hydrolyse mit teildenaturiertem Enzym (69\% Restaktivität) keine anomale Temperaturabhängigkeit auf.
\end{abstract}

Wie in der vorangehenden Mitteilung * gezeigt werden konnte, erhält man bei der Hydrolyse von Tributyrin durch Pankreaslipase für den Temperaturverlauf der Hydrolysengeschwindigkeit im $\lg v_{\mathrm{br}} / 1 / T$-Diagramm keine Gerade, sondern eine Kurve. Ziel dieser Untersuchungen sollte es sein, das aufgetretene anomale Temperaturverhalten genauer zu prüfen und Einflüsse auf seinen Verlauf kennen zu lernen.

\section{Experimentelles}

Versuchsaufbau. Bei sämtlichen in dieser Arbeit angeführten Versuchen wurde die Hydrolyse der Glycerinester potentiometrisch ohne Puffer, jedoch bei konst. $p_{\mathrm{H}}$-Wert verfolgt. Es fand dabei die bereits von uns an anderer Stelle ${ }^{7}$ beschriebene automatisch arbeitende Apparatur Verwendung.

Enzympräparate. Die bezogenen, gereinigten Schweinepankreas-Lipasepräparate (Calbiochem bzw. Serva) wurden zur weiteren Anreicherung in bidest. Wasser

* I. Z. Naturforschg. 22 b, 524 [1967]. bei $0{ }^{\circ} \mathrm{C}$ gelöst $(12,5 \mathrm{mg}$ in $25 \mathrm{ml})$, von ungelösten $\mathrm{An}$ teilen, die ebenfalls lipolytische Eigenschaften aufwiesen, abzentrifugiert und bei $0{ }^{\circ} \mathrm{C}$ aufbewahrt $\left(p_{\mathrm{H}} 6,3\right)$. $1 \mathrm{ml}$ Enzymlösung enthielt durchschnittlich 0,1 bis $0,15 \mathrm{mg}$ Eiweiß (Biuret); die spez. Aktivität betrug $1-1,1$ E.E.mg ${ }^{-1}$ (Triacetin, $p_{\mathrm{H}} 6,25,25{ }^{\circ} \mathrm{C}$ ), die Enzymkonzentration in den Reaktionslösungen $2 \mathrm{mg} l^{-1}$. Substrate. Sowohl das verwendete Tributyrin (TBU; rein, Merck), als auch das Triacetin (TAC; Riedel de Haën) wurden zusätzlich im Vacuum fraktioniert.

Bestimmung der Fehlerabweichung. Bei der Überlegung, welche Parameter unter den geschilderten Versuchsbedingungen die Reaktionsgeschwindigkeit wesentlich beeinflussen, sind vor allem die Faktoren zu berücksichtigen, die Fehler in der Enzymmenge bewirken.

Während einer Versuchsreihe, die sich in der Regel über 8-10 Stdn. erstreckte, nahm die Aktivität des bei $0{ }^{\circ} \mathrm{C}$ gelagerten Enzyms durchschnittlich um 2,5\% ab; dies bedeutet, daß man den Aktivitätsverlust innerhalb 45 Min., dem Zeitintervall zweier Bestimmungen, vernachlässigen kann. Für das Pipettieren von $0,5 \mathrm{ml}$ Enzymlösung ergab sich der experimentell ermittelte Fehler von $\pm 0,5 \%$, für die Auswertung der vom Auto-

7 F. Pаtat u. G. TAlsky, Z. Naturforschg. 22 b, 524 [1967]. 